\title{
Interconnection Between Metabolism and Cell Cycle in Cancer
}

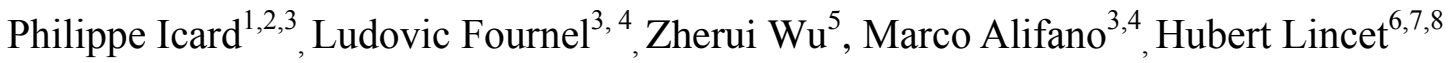

${ }^{1}$ CHU de Caen, Université Caen Normandie, Medical School, Caen, F-14000, France

${ }^{2}$ INSERM U1086, BioTICLA axis, Université Caen Normandie, F-14000, France

${ }^{3}$ Department of Thoracic Surgery, Paris Center University Hospital, AP-HP, Paris, France

${ }^{4}$ Paris Descartes University, Paris, France

${ }^{5}$ INSERM UMRS 1007, Paris Descartes University, 75270 Paris cedex 06, France

${ }^{6}$ Inserm U1052, CNRS UMR5286, Centre de Recherche en Cancérologie de Lyon (CRCL), France

${ }^{7}$ Université Lyon Claude Bernard 1, Lyon, France

${ }^{8}$ ISPB, Faculté de Pharmacie, Lyon, France

*Corresponding author: philippe.icard@aphp.fr

Keywords: cell cycle; glycolysis; PKM2; PFKFB3; D cyclin; GAPDH 


\begin{abstract}
Cell cycle progression and division is regulated by check-point controls and sequential activation of cyclin-dependent kinases (CDK). Understanding how these events occur in synchrony with metabolic changes could have important therapeutic implications. For biosynthesis, cancer cells enhance glucose and glutamine consumption. Inactivation of PKM2 promotes transcription in G1 phase. Glutamine metabolism supports DNA replication in S phase and lipids synthesis in G2 phase. A boost in glycolysis and oxidative metabolism can temporarily furnish more ATP when necessary (G1/S transition, segregation of chromosomes). Recent studies have shown that a few metabolic enzymes (PKM2, PFKFB3, GAPDH) also periodically translocate to the nucleus and oversee cell cycle regulators /or oncogene expression (c-Myc). Targeting these metabolic enzymes could increase the response to CDK inhibitors.
\end{abstract}




\section{The Cell Cycle and the Warburg Effect}

Cell cycle progression is orchestrated by sequential activation of cyclin-dependent kinases (CDKs) by their proper cyclin partner [1]. Activated CDKs phosphorylate RNA-polymerase II ensuring transcription of proteins sustaining biosynthesis which requires an energy supply produced by oxidative phosphorylation (OXPHOS) and/or glycolysis. Cancer cells often display enhanced aerobic glycolysis (lactate production even in the presence of oxygen), a metabolism referred as the "Warburg effect" which supports their proliferation and aggressiveness [2]. In this review, we aimed to describe how this metabolism is interconnected with cell cycle progression through reciprocal activation of metabolic enzymes and cell regulators. Understanding this linkage may help in the development of new anticancer strategies, and improve treatments to finally overcome drug resistance.

\section{The Warburg Effect}

The Warburg effect favors proliferation, invasiveness and resistance to apoptosis [2, 3]. This reprogramming metabolism is promoted by hypoxia and hypoxia inducible factor-1 (HIF-1), c-Myc and various oncogenes promoting aggressive phenotypes such as K-ras driven lung, colonic or pancreatic cancers, and ErbB2-driven breast cancers $[4,5]$. The Warburg effect is related to a shift from oxidative to reductive metabolism related to the inhibition of pyruvate dehydrogenase (PDH) by pyruvate dehydrogenase kinase 1 (PDK1) and the inhibition of complex IV of the respiratory chain related to p53-deficiency [6] or the Crabtree effect [7] (see glossary).

PDK1 is activated by HIF-1 [8] and two kinases (AKT [9] and the multifunctional enzyme phosphoglycerate kinase 1 (PGK1) [10]) previously activated by K-Ras which translocate into 
the mitochondria. The downregulation of mitochondria limits toxic reactive oxygen species (ROS) and avoids the negative feedback exercised by high ATP and citrate production on phosphofructokinase1 (PFK1), the main enzyme regulating glycolysis (Figure 1). The reduced mitochondrial production of ATP and $\mathrm{CO}_{2}$ (two major sources of $\mathrm{H}^{+}$) favors the establishment of an alkaline intracellular $\mathrm{pH}(\mathrm{pHi})$ (around 7.4) also maintained by up-regulated membrane exchangers expelling $\mathrm{H}^{+}$into microenvironment $[9,10]$. This $\mathrm{pHi}$ condition sustains proliferation by various processes [2] such as enhancement of PFK1 activity, histone acetylation [11], microtubule polymerization in prophase [12], inactivation of p53 and inhibition of apoptosis [13], and multi drug resistance (MDR) [10]. Moreover, acidification of the microenvironment promotes invasion, angiogenesis and immune tolerance [8-10, 14].

\section{Cell Cycle Progression}

Cell cycle is an irreversible process which sustains an ordered sequence of events controlled by three main checkpoints (Box 1). The complex mechanisms which regulate cell cycle have been extensively studied in yeast and mammalian cells, and more than 20 cyclin-dependent kinases (CDKs) have been identified $[1,15]$. Among them, CDK1, 2, 4 and 6 play a major role in cell cycle progression, a process highly disturbed in cancer cells [1, 16-19]. The activation of CDKs depends on synthesis and degradation of their regulatory cyclins. Growth factors initiate entrance in G1 phase and induce activation of cyclins D (D1, D2 and D3) which interact with CDK4 or CDK6. Increased cyclin D-CDK4/6 activity results in phosphorylation of the retinoblastoma protein $(\mathrm{pRB})$ and inactivation of $\mathrm{p} 53$, inducing the release of E2F from pRB-E2F complex. This release leads to inactivation of the restriction G1 checkpoint that arrests the cell cycle when a low availability in nutrients is detected.

Activation of cyclin E-CDK2 enables transcription of genes required for entrance into S phase (Figure 2A). Cyclin A-CDK2 drives progression in S, cyclin A-CDK1 in G2 phase, and cyclin 
B-CDK1 regulates progression from late G2 until exit of mitosis [1, 17]. Other kinases, in particular Polo-like Kinase 1 (PLK1) and Aurora kinase A/B are critical for progression through mitosis, chromosome segregation, and cytokinesis [19].

This irreversible progression of cell cycle is due to the gradual accumulation and abrupt destruction of cyclins at specific times by two E3 proteasomal ligases, APC/C (Anaphasepromoting complex/cyclosome) and SCF (Skp1/ Cullin/F-box) [1, 17, 20] (Figure 2B). CDK activities are also inhibited by two families of inhibitors (CKIs), in particular the INK4 family (p16, p15, p18 and p19) which bind specifically to CDK4 and CDK6, and the WAF1/CIP/KIP family (p21, p27 and p57) which inhibit all major $\mathrm{CDK}_{\mathrm{S}}$. Moreover, CDKs can be inhibited by phosphorylation, in particular CDK1 phosphorylated by Wee1 and Myt1, suspending entrance in mitosis. This CDK1 inactivation is reversed by Cdc25 phosphatase and SCF- $\beta$ TrCP that degrade Wee1 [1, 19].

Cancer cells lose many of these inhibitory controls because of the inactivation or mutation of suppressor genes, and overexpression or amplification of oncogenes, all alterations resulting in aberrant transcription with upregulation of cyclins-CDKs, leading to uncontrolled cell cycle progression and mitosis.

\section{The Interconnection between the Cell Cycle and Metabolism}

All ten glycolytic enzymes demonstrate non-metabolic functions sustaining cancer proliferation, aggressiveness, and metastatic potential [21, 22]. Several of them periodically translocate into the nucleus, linking metabolism with cell cycle progression. As cyclins, the activities of these enzymes oscillate because they are degraded by similar ubiquitin proteasomal complexes [20]. 
G1 Phase: Intense Protein Synthesis

In G1, active synthesis of thousands of proteins (entering the ribosome machinery, in particular) and hundreds of enzymes occurs to duplicate the biomass [23]. Histone acetylation opens chromatin for DNA transcription. Cyclin D-CDK4 activates acetyltransferases (HATs) such as GCN5 (general control non-repressed protein 5) [24], a factor repressing nuclear respiratory factor 1 [25] and inhibiting mitochondrial activity through acetylation of PPAR $\gamma$ PGC-1 $\alpha$ (peroxisome proliferator activated receptor $\gamma$ coactivator-1 $\alpha$ ) [26]. The E2F1-CDK4 axis also represses PGC-1 $\alpha$ and OXPHOS gene expression while it inactivates PDH [27, 28]. Furthermore, the downregulation of mitochondria promotes an alkaline pHi favoring histone acetylation [11]. Acetyl-CoA is oriented towards histone acetylation because the accumulation of cyclin D downregulates lipid synthesis [29]. Acetyl-CoA also feeds the hexosamine pathway which could serve as sensor of nutrient levels because it consumes glucose and glutamine (Figure 1). Glucosamine biosynthesis could also play an important role in many aspects of cancer development (epigenetics, transcription, signaling, DNA repair, proteins folding, maturation and trafficking) $[16,30]$.

Acetyl-CoA can be furnished by several sources [2]: (i) the cytoplasmic and/or nuclear activity of ATP-citrate lyase (ACLY) transforming citrate into oxaloacetate (OAA) and acetyl-CoA [31]; the citrate being derived from mitochondrial synthesis or from cytosolic carboxylation of $\alpha$-ketoglutarate ( $\alpha$-keto); (ii) the nuclear activity of acetyl-CoA synthase (ACS1) using acetate as substrate [32]; and (iii) the nuclear activity of PDH translocating from mitochondria to the nucleus during G1 and S phases [33].

Methylation of the genome and epigenome is sustained by the activity of the serinemethionine pathway producing methyl groups also involved in other syntheses such as glutathione, $\alpha$-ketoglutarate ( $\alpha$-keto), and polyamines [3, 34, 35]. These consumptions likely favor a global state of genome demethylation altering gene expression, whereas local areas of 
hypermethylation inactivate suppressor genes $[2,36]$. Mutations of enzymes involved in tricarboxylic acid cycle (TCA) lead to the accumulation of molecules [succinate, fumarate, and 2-hydroxyglutarate (2HG)] promoting demethylase functioning [3].

Hexokinase 2 (HK2) sustains a high glycolytic phenotype because its attachment to the outer mitochondrial membrane prevents retrograde inhibition by the high level of glucose-6phosphate (G6P) and thus favors glucose uptake by membrane glucose transporter1 (GLUT1). Moreover, this HK2 attachment provides rapid access to ATP synthesized by mitochondria and would prevent apoptosis [37]. HK2 inhibition leads to dephosphorylation of Bad, causing the translocation of the pro-apoptotic factor Bax to the mitochondria, thus, activating the release of cytochrome and apoptosis [38]. It is tempting to speculate, that like glucokinase (HK4) [39], HK2 resides in the same mitochondrial complex that Bad, and this proximity results in Bad inactivation.

HK2 is activated by CDK2 in cancer-associated fibroblasts (CAFs) promoting aerobic glycolysis in these cells, a metabolism which can support oxidative functioning in cancer cells through a reverse Warburg effect $[40,41]$.

PFK1 is a main regulator of glycolysis which transforms fructose-6 phosphate (F6P) into fructose-1,6-bisphosphate (F1,6P). It is linked to the IGF-1R/PI3K/AKT proliferative pathway by fructose-1,6-bisphosphate $(\mathrm{F} 1,6 \mathrm{P})$ which stimulates Ras through activation of its strong regulator Son of sevenless homolog 1 (Sos1), the mammalian ortholog of yeast Cdc25 [42]. Thus, in many cancer cells, a vicious circle is created between PFK1, Ras, and the proliferative PI3K-AKT pathway, resulting in upregulation (expression and activation) of GLUT1 and many glycolytic enzymes, in particular those related to the activation of cyclins, such as embryonic pyruvate kinase M2 (PKM2) [21, 43].

The tetrameric form of PKM2 converts phosphoenolpyruvate (PEP) into pyruvate sustaining lactate production by lacticodehydrogenase-5 (LDH-5). In contrast, the dimeric inactive form 
of PKM2 creates 'a bottle neck' at the end of glycolysis shifting metabolites towards biosynthesis. The balance between tetrameric and dimeric forms is allosterically regulated: PKM2 is activated by F1,6BP, and serine, and is inversely inactivated by high concentrations of ATP and alanine [44].

In G1 phase, monomeric PKM2 translocates into the nucleus [45] increasing the expression of c-Myc and promoting $\beta$-catenin transactivation leading to upregulation of CCND1, the gene encoding cyclin D1 [46] (Figure 2C).

These nuclear functions of PKM2 would result from PKM2 dephosphorylation by the phosphatase Cdc25A [47]. c-Myc promotes in turn expression of several glycolytic genes (in particular GLUT1, PKM2 and LDHA), while it concomitantly activates CDC25A gene expression. Thus, the phosphatase Cdc25A upregulates CDC25A gene expression in a positive feedback loop [47]. Finally, PKM2 translocation results in concomitant activation of the Warburg effect, c-Myc expression and cell cycle progression.

As a protein kinase, PKM2 also activates the signal transducer and activator of transcription 3 (STAT3) and phosphorylates histone $\mathrm{H} 3$ promoting active transcription of genes such as HIF1, STAT3, and c-Myc [21, 44, 47]. As a result, PKM2 which is activated by proliferative signaling such as EGFR, nuclear factor kappa B (NFkB), and AKT [44], sustains cell cycle progression and active transcription of glycolytic enzymes and glutamine synthase1 (GLS1) $[5,48]$.

\section{G1/S Transition Requires an 'Energy Boost'}

PFK2, also called PFKFB (6-phosphofructo-2-kinase/fructose-2,6-biphosphatase), is activated in late G1 phase to enhance glycolysis at the restriction period control, allowing further G1 /S transition. The inactivation of APC/C-Cdh1 in mild G1 to early S phase, releases the 'break' 
exercised on PFKFB3 and D-cyclins [17, 20] resulting in enhancement of glycolysis and glutaminolysis in this highly nutrient sensitive period. Among the four mammalian bidirectional PFKFB isoenzymes, PFKFB3 has the highest kinase/phosphatase activity ratio (740/1), producing fructose-2,6-biphosphate (F2,6BP), a strong activator of PFK1, which directs the carbon flux towards glycolysis [49]. Overexpressed in many cancers, especially aggressive ones, PFKFB is induced by HIF-1 and AKT. Loss or dysfunction of p53 increases PFKFB3 activity through p53-inducible regulator of glycolysis and apoptosis (TIGAR) inhibition release [13].

PFKFB3 is degraded at the onset of S phase by SCF- $\beta$-TrCP $[17,20]$. During its brief period of activation, PFKFB3 traffics to the nucleus where its product F2,6BP represses p27Kip1, a potent inhibitor of cyclins D and E, while it activates cyclin D3, all processes resulting in late G1 phase progression and G1/S transition [49]. Cyclin D3 induces mitochondrial activity through PPAR $\gamma$ activation [50], a process reinforced by GCN5 inhibition by active E2F1 and c-Myc [51]. Notably, transient mitochondrial fusion can occur at this time, generating more ATP for E2F activation and entry in S phase [52]. PFKFB3 also favors progression and entrance to mitosis by increasing expression of CDK1 and Cdc25 [49].

\section{S Phase Progression: Replication of DNA}

Activation of cyclins E and A by E2F results in the transcription of various genes involved in DNA replication such as thymidine kinase and DNA polymerase [28]. Re-entrance to G1 phase is likely prevented by the inhibition of HK2 by the high level of cyclin D1, a regulation reducing glucose uptake at the onset of S phase [29].

As depicted in Figure 1, glycolysis-branched pathways [the pentose phosphate pathway (PPP) and the serine-methionine pathway] and glutaminolysis, provide various molecules (ribose 5- 
phosphate, serine, methyl groups, glutamate, aspartate) that are involved in the biosynthesis of nucleotides, and acetyl-CoA sustaining lipid synthesis [34, 44, 53].

Cyclin D3/CDK6 promotes functioning of the oxidative branch of PPP by inhibiting PFK1 and PKM2 [50]. Accordingly, activity of glucose-6-phosphate dehydrogenase (G6PDH) increases in late $\mathrm{G} 1[54,55]$. Notably, due to epigenetic reprogramming, metastatic pancreatic ductal adenocarcinoma cells would preferentially rely on the oxidative branch of PPP regulated by G6PDH [56], in contrast to most primary cancer cells (KRAS-mutated in particular) mainly relying on the non-oxidative branch [57]. This pathway is regulated by transketolase1 (TKL1) which is detected in late G1 and increases during S-phase [55, 58]. The functioning of non-oxidative PPP is favored by inactivation of TIGAR directing the carbon flux in the glycolytic direction because this inactivation promotes PFK1 activity [59]. Aldolase (ALDO) catalyzes the reversible conversion of F1,6P to glyceraldehyde3-phosphate (G3P) and dihydroxyacetone phosphate (DHAP). Its activity is promoted by glutaminolysis and lactate, and its nuclear translocation correlates with AKT phosphorylation, cell proliferation, and DNA replication [60].

Triosephosphate isomerase (TPI) converts glyceraldehyde-3-phosphate (G3P) into DHAP. TPI is inhibited by phosphoenolpyruvate (PEP) which accumulates upstream dimeric PKM2 [53] and by cyclin A-CDK2, the complex sustaining S phase progression [61]. TPI inhibition promotes PPP functioning.

GLS1 regulates glutamine breakdown metabolism. It remains highly activated during S phase because unlike PFKFB3, it is insensitive to degradation by SCF- $\beta$-TrCP. Indeed, GLS1 is degraded in late mitosis by APC/C-Cdh1 [17, 20].

Glutamine breakdown and oxidation increases in S are stimulated by c-Myc [5, 48], providing molecules and ATP for DNA polymerase functioning. The increasing production of ROS, 
toxic for DNA replication, must be neutralized by reduced glutathione molecules requiring regeneration of NADPH, $\mathrm{H}^{+}$molecules. This cofactor is regenerated by the oxidative part of PPP and/or functioning of cytosolic enzymes sustained by glutaminolysis, such as malate dehydrogenase, isocitrate dehydrogenase (IDH), and malic enzyme (ME) [2, 3]. NADPH, $\mathrm{H}^{+}$ production also sustains lipid synthesis, while R5P is recycled back to glycolysis by TKL1 or transformed in acetyl-CoA by transketolase-like 1 (TKTL1) [55, 58].

\section{G2 Phase: Membrane Formation and Chromatin Compaction}

Cancer cells enhance their de novo lipid synthesis during S phase and G2 phase, this synthesis being promoted by E2F, cyclin D3, and low molecular weight isoforms of cyclin E (LMW-E) associating with ACLY sustaining acetyl-CoA production $[27,28]$. The drop of cyclin D1 releases the inhibition of acetyl-CoA carboxylase (ACC) and fatty acid synthase (FAS) [23, 29, 62]. Glyceraldehyde 3-phosphate dehydrogenase (GAPDH) stimulates the functioning of end-part glycolysis in late interphase. Its activity promotes those of PGK1 and PKM2 producing ATP, while LDH-5 regenerates NAD ${ }^{+}$for GAPDH functioning. PKM2 activation releases the break exercised by PEP on TPI: the synthesis of DHAP is activated, sustaining that of glycerol phosphate required for triglycerides formation [53].

Almost undetectable in G1, the nuclear concentration of GAPDH increases from S phase to G2/M. Over-expressed in many tumors, GAPDH accelerates cell cycle progression by inducing an advancement of the cyclin B-CDK1 peak [63] and delays degradation of telomeres [64]. This multifunctional enzyme is also involved in DNA repair and apoptosis resistance $[22,65]$ and in preservation of a contingent of intact mitochondria by enhancing mitophagy [65]. 
Histone deacetylation is required for transcriptional repression and chromatin compaction before $\mathrm{M}$ phase entry. It is favored by intracellular acidity that increased during $\mathrm{S}$ phase due to glutamine oxidation. When pHi goes below approximately 7.0, net acetate flow reverses and goes outward to chromatin neutralizing the too high proton concentration $[10,11,66]$. Histone deacetylation is also regulated by sirtuin 1 (SIRT1) dependent NAD ${ }^{+}$, a lack of this cofactor altering this process [67].

\section{Mitosis}

Cyclin B-CDK1 governs mitotic entry progression with AURKA and PLK1 [19, 68]. CDK1 is inactivated by Wee1 and Myt1 kinases [1, 19] and activated by phosphatase Cdc25 [69]. In prophase, biosynthetic activities continue and stop thereafter. The occurrence of mitochondrial fission is regulated by CDK1-cyclin B and AURKA which promote activity of dynamin related protein 1 (Drp1) [70, 71]. Impaired mitochondrial fission can lead to dysfunctional energy production and unequal distribution of the organelle into each daughter cell [71]. The downregulation of mitochondria favors a return to an alkaline pHi which seems an essential condition for microtubule polymerization around the centrosome [12].

The metaphase checkpoint ensures that all chromosomes are properly attached to the mitotic spindle before the switch to anaphase. Degradation of securin and cyclin B by APC/C-Cdh20 liberates separase, triggering the disjunction of sister chromatids and their migration in anaphase [72]. PKM2 participates in this segregation regulation by acting as a protein kinase that controls the fidelity of separated chromosomes [73]. In response to an increasing energy demand required by this checkpoint, cyclin B1-CDK1 can phosphorylate complex I of the respiratory chain generating ATP, in particular for the functioning of 'motor' proteins (dynein and kinesin) which move chromosomes [74]. This transient activation of mitochondria likely 
promotes acidic pHi which favors microtubule disassembly [12].

The transitions to telophase and mitosis exit are allowed by the inactivation of cyclin B by APC/C-Cdc20 and APC/C-Cdh1 liberating CDK1 [72]. In yeast, cell division occurs when oxygen consumption and acetyl-CoA level significantly decrease $[75,76]$. A similar process probably occurs in mammals: the decrease or arrest of mitochondrial activity in telophase promotes an alkaline $\mathrm{pHi}$ in each daughter cell. As we have seen, this $\mathrm{pH}$ condition favors histone acetylation [11], and DNA transcription while a new G1 phase is reengaged.

\section{Concluding Remarks and Future Perspectives}

The metabolism of cancer cells raises a multitude of issues regarding its mechanisms and causes and its universality and inhomogeneity within various cancer cell types (see Outstanding Questions). The development of metabolic strategies should be accompanied in the future by methods and biomarkers capable of selecting patient profiles eligible for metabolic treatment enhancing the effects of chemotherapies, in particular of the cell cycle inhibitors.

As we have seen, the Warburg effect allows flexibility and adaptability of cancer cells to various environmental conditions. This plasticity selects diverse cell phenotypes more or less proliferative, sensitive, or resistant to hypoxia and current treatments $[2,10,14]$. Poorly differentiated phenotypes are promoted by the Warburg effect [2] and the inactivation of mitochondria is also favored by inhibition of PGC- $1 \alpha \mathrm{c}$ by inactive SIRT1/3 [67, 77-79], loss of suppressive controls exercised by p53 [6, 59], factor forkhead box protein O1 (FOXO1) and AMP-activated protein kinase (AMPK) [80, 81]. Importantly, some cell-clones are able to switch from reductive to oxidative metabolism (and back again), a flexibility which confers on them a metabolic advantage increasing their resistance and/or metastatic potential $[56,77$, 
82, 92]. Various metabolic and epigenetic phenotypes are selected and supported by the oscillations of key metabolites, nicotinamide cofactors and ATP; their concentration fluctuations likely promote genomic instability, DNA alterations, and chromosome damages. For example, a lack of $\mathrm{NAD}^{+}$promotes inactivation of SIRT favoring mitochondrial downregulation and histone deacetylation, while it may increase DNA damage by altering PARP1 (poly (ADP-ribose) polymerase1) functioning [78, 79]. As we now understand, the sequential translocations to the nucleus of key metabolic enzymes (PKM2, PFKFB3, ALDO, GAPDH) create vicious circles linking metabolism with cell cycle regulators and oncogenes (in particular K-ras and c-Myc). And finally, the cell cycle can be viewed as sequential oscillations of cyclins-CDKs, glycolytic enzymes, and metabolites, whose variations rule gene expression and interconnect biosynthesis and cell cycle progression.

Understanding how the metabolic events occur synchronously with cell cycle progression can be a source of inspiration for developing new anti-cancer strategies. The high dependence on glycolysis of many cancer cells can constitute the Achille's heel. However, some cell clones can develop alternative pathways to shunt enzymatic inhibitions, or may use OXPHOS [92] or autophagy to survive [84]. The glycolytic pathway constitute the 'main street' that could be targeted by inhibitors of regulatory enzymes linked thermodynamically with ATP consumption (HK2, PFK1, PFKFB3) or with ATP production (PGK1, PKM2), or by "paninhibitor" such as 3-bromopyruvate targeting several enzymes of glycolysis [88]. Clearly, GLS1 sustaining S phase could also be targeted [86]. Considering that PFKFB3, PKM2 and GAPDH activate cell cycle progression by non-glycolytic functions, these enzymes appear as preferential targets for inhibitors, that could be used in association with CDK inhibitors recently listed $[15,87]$. As example, inhibition of PKM2 or PFKFB3 could reinforce the effect of selective inhibition of CDK4/6, while inhibition of GAPDH (but also of ACLY and FAS) could be tested in association with selective inhibition of CDK1, AURK, or PLK1 
kinases. Inhibitors of PFKFB3 or GLS1 could also increase the response to CDK pan-

inhibitors. However, beside these enzymatic inhibitions, other metabolic strategies could be addressed to inhibit or reverse the metabolism supporting growth (Box 2). Finally, testing these metabolic approaches with current available therapies targeting proliferative pathways, nucleotide synthesis and cyclin-CDKs, is certainly warranted.

\section{Acknowledgements}

The authors thank Oriane Rein Dubuisson for her technical assistance in the realization of figures.

The Authors regret that due to space limitations that they were unable to cite many excellent papers that shaped our modern understanding of cell cycle and cancer cells metabolism.

\section{Conflicts of Interest}

Authors disclose no conflicts of interest

\section{References}

1. Malumbres, M. (2014) Cyclin-dependent kinases. Genome Biol. 15 (6), 122.

2. Icard, P. et al. (2018) How the Warburg effect supports aggressiveness and drug resistance of cancer cells? Drug Resist Updat 38, 1-11.

3. Vander Heiden, M.G. and DeBerardinis, R.J. (2017) Understanding the Intersections between Metabolism and Cancer Biology. Cell 168 (4), 657-669.

4. Cairns, R.A. (2015) Drivers of the Warburg phenotype. Cancer J 21 (2), 56-61.

5. Dang, C.V. (2016) A Time for MYC: Metabolism and Therapy. Cold Spring Harb Symp Quant Biol 81, 79-83.

6. Matoba, S. et al. (2006) p53 regulates mitochondrial respiration. Science 312 (5780), 16501653.

7. Diaz-Ruiz, R. et al. (2011) The Warburg and Crabtree effects: On the origin of cancer cell energy metabolism and of yeast glucose repression. Biochim Biophys Acta 1807 (6), 568-76. 8. Semenza, G.L. (2013) HIF-1 mediates metabolic responses to intratumoral hypoxia and oncogenic mutations. J Clin Invest 123 (9), 3664-71. 
9. Corbet, C. and Feron, O. (2017) Tumour acidosis: from the passenger to the driver's seat. Nat Rev Cancer 17 (10), 577-593.

10. White, K.A. et al. (2017) Cancer cell behaviors mediated by dysregulated $\mathrm{pH}$ dynamics at a glance. J Cell Sci 130 (4), 663-669.

11. McBrian, M.A. et al. (2013) Histone acetylation regulates intracellular pH. Mol.Cell 49

(2), 310-321.

12. Gagliardi, L.J. and Shain, D.H. (2013) Is intracellular pH a clock for mitosis?

Theor.Biol.Med.Model. 10, 8.

13. Lee, P. et al. (2014) TIGAR, TIGAR, burning bright. Cancer Metab 2 (1), 1.

14. Leung, E. et al. (2017) Metabolic targeting of HIF-dependent glycolysis reduces lactate, increases oxygen consumption and enhances response to high-dose single-fraction radiotherapy in hypoxic solid tumors. BMC Cancer 17 (1), 418.

15. Whittaker, S.R. et al. (2017) Inhibitors of cyclin-dependent kinases as cancer therapeutics. Pharmacol Ther 173, 83-105.

16. Kaplon, J. et al. (2015) Two-way communication between the metabolic and cell cycle machineries: the molecular basis. Cell Cycle 14 (13), 2022-32.

17. Moncada, S. et al. (2012) Fulfilling the metabolic requirements for cell proliferation.

Biochem.J 446 (1), 1-7.

18. Kalucka, J. et al. (2015) Metabolic control of the cell cycle. Cell Cycle 14 (21), 3379-88.

19. Aarts, M. et al. (2013) Tumour selective targeting of cell cycle kinases for cancer treatment. Curr Opin Pharmacol 13 (4), 529-35.

20. Tudzarova, S. et al. (2011) Two ubiquitin ligases, APC/C-Cdh1 and SKP1-CUL1-F (SCF)beta-TrCP, sequentially regulate glycolysis during the cell cycle. Proc.Natl.Acad.Sci.U.S.A 108 (13), 5278-5283.

21. Lu, Z. and Hunter, T. (2018) Metabolic Kinases Moonlighting as Protein Kinases. Trends Biochem Sci 43 (4), 301-310.

22. Lincet, H. and Icard, P. (2015) How do glycolytic enzymes favour cancer cell proliferation by nonmetabolic functions? Oncogene 34 (29), 3751-9.

23. Buchakjian, M.R. and Kornbluth, S. (2010) The engine driving the ship: metabolic steering of cell proliferation and death. Nat.Rev.Mol.Cell Biol. 11 (10), 715-727.

24. Lee, Y. et al. (2014) Cyclin D1-Cdk4 controls glucose metabolism independently of cell cycle progression. Nature 510 (7506), 547-551.

25. Wang, C. et al. (2006) Cyclin D1 repression of nuclear respiratory factor 1 integrates nuclear DNA synthesis and mitochondrial function. Proc Natl Acad Sci U S A 103 (31), 11567-72.

26. Dominy, J.E., Jr. et al. (2010) Nutrient-dependent regulation of PGC-1alpha's acetylation state and metabolic function through the enzymatic activities of Sirt1/GCN5.

Biochim.Biophys.Acta 1804 (8), 1676-1683.

27. Lopez-Mejia, I.C. and Fajas, L. (2015) Cell cycle regulation of mitochondrial function. Curr Opin Cell Biol 33, 19-25.

28. Escote, X. and Fajas, L. (2015) Metabolic adaptation to cancer growth: from the cell to the organism. Cancer Lett 356 (2 Pt A), 171-5.

29. Sakamaki, T. et al. (2006) Cyclin D1 determines mitochondrial function in vivo. Mol.Cell Biol. 26 (14), 5449-5469.

30. Hanover, J.A. et al. (2018) O-GlcNAc in cancer: An Oncometabolism-fueled vicious cycle. J Bioenerg Biomembr 50 (3), 155-173.

31. Wellen, K.E. et al. (2009) ATP-citrate lyase links cellular metabolism to histone acetylation. Science 324 (5930), 1076-1080.

32. Schug, Z.T. et al. (2016) The metabolic fate of acetate in cancer. Nat Rev Cancer 16 (11), 708-717. 
33. Sutendra, G. et al. (2014) A nuclear pyruvate dehydrogenase complex is important for the generation of acetyl-CoA and histone acetylation. Cell 158 (1), 84-97.

34. Locasale, J.W. (2013) Serine, glycine and one-carbon units: cancer metabolism in full circle. Nat Rev Cancer 13 (8), 572-83.

35. Icard, P. et al. (2014) The metabolic cooperation between cells in solid cancer tumors.

Biochim Biophys Acta 1846 (1), 216-25.

36. Plass, C. et al. (2013) Mutations in regulators of the epigenome and their connections to global chromatin patterns in cancer. Nat Rev Genet 14 (11), 765-80.

37. Mathupala, S.P. et al. (2009) Hexokinase-2 bound to mitochondria: cancer's stygian link to the "Warburg Effect" and a pivotal target for effective therapy. Semin Cancer Biol 19 (1), 1724.

38. Xu, R.H. et al. (2005) Inhibition of glycolysis in cancer cells: a novel strategy to overcome drug resistance associated with mitochondrial respiratory defect and hypoxia. Cancer Res. 65 (2), 613-621.

39. Danial, N.N. et al. (2003) BAD and glucokinase reside in a mitochondrial complex that integrates glycolysis and apoptosis. Nature 424 (6951), 952-956.

40. Hu, J.W. et al. (2014) Hexokinase 2 regulates G1/S checkpoint through CDK2 in cancerassociated fibroblasts. Cell Signal 26 (10), 2210-6.

41. Wilde, L. et al. (2017) Metabolic coupling and the Reverse Warburg Effect in cancer: Implications for novel biomarker and anticancer agent development. Semin Oncol 44 (3), 198-203.

42. Peeters, K. et al. (2017) Fructose-1,6-bisphosphate couples glycolytic flux to activation of Ras. Nat Commun 8 (1), 922.

43. Courtnay, R. et al. (2015) Cancer metabolism and the Warburg effect: the role of HIF-1 and PI3K. Mol Biol Rep 42 (4), 841-51.

44. Prakasam, G. et al. (2018) Posttranslational Modifications of Pyruvate Kinase M2:

Tweaks that Benefit Cancer. Front Oncol 8, 22.

45. Lv, L. et al. (2013) Mitogenic and oncogenic stimulation of K433 acetylation promotes PKM2 protein kinase activity and nuclear localization. Mol Cell 52 (3), 340-52.

46. Yang, W. and Lu, Z. (2013) Nuclear PKM2 regulates the Warburg effect. Cell Cycle 12 (19), 3154-8.

47. Liang, J. et al. (2016) PKM2 dephosphorylation by Cdc25A promotes the Warburg effect and tumorigenesis. Nat Commun 7, 12431.

48. Bott, A.J. et al. (2015) Oncogenic Myc Induces Expression of Glutamine Synthetase through Promoter Demethylation. Cell Metab 22 (6), 1068-77.

49. Yalcin, A. et al. (2009) Nuclear targeting of 6-phosphofructo-2-kinase (PFKFB3)

increases proliferation via cyclin-dependent kinases. J.Biol.Chem. 284 (36), 24223-24232.

50. Wang, H. et al. (2017) The metabolic function of cyclin D3-CDK6 kinase in cancer cell survival. Nature 546 (7658), 426-430.

51. Yin, Y.W. et al. (2015) The Histone Acetyltransferase GCN5 Expression Is Elevated and Regulated by c-Myc and E2F1 Transcription Factors in Human Colon Cancer. Gene Expr 16 (4), 187-96.

52. Mitra, K. et al. (2009) A hyperfused mitochondrial state achieved at G1-S regulates cyclin E buildup and entry into S phase. Proc.Natl.Acad.Sci.U.S.A 106 (29), 11960-11965.

53. Gruning, N.M. et al. (2014) Inhibition of triosephosphate isomerase by phosphoenolpyruvate in the feedback-regulation of glycolysis. Open Biol 4, 130232.

54. Jiang, P. et al. (2014) Regulation of the pentose phosphate pathway in cancer. Protein Cell 5 (8), 592-602.

55. Diaz-Moralli, S. et al. (2013) Targeting cell cycle regulation in cancer therapy.

Pharmacol.Ther. 138 (2), 255-271. 
56. McDonald, O.G. et al. (2017) Epigenomic reprogramming during pancreatic cancer progression links anabolic glucose metabolism to distant metastasis. Nat Genet 49 (3), 367 376.

57. Ying, H. et al. (2012) Oncogenic Kras maintains pancreatic tumors through regulation of anabolic glucose metabolism. Cell 149 (3), 656-70.

58. Diaz-Moralli, S. et al. (2016) A key role for transketolase-like 1 in tumor metabolic reprogramming. Oncotarget 7 (32), 51875-51897.

59. Gomes, A.S. et al. (2018) p53 and glucose metabolism: an orchestra to be directed in cancer therapy. Pharmacol Res 131, 75-86.

60. Mamczur, P. et al. (2013) Nuclear localization of aldolase A correlates with cell proliferation. Biochim Biophys Acta 1833 (12), 2812-2822.

61. Lee, W.H. et al. (2010) Functional inactivation of triosephosphate isomerase through phosphorylation during etoposide-induced apoptosis in HeLa cells: potential role of Cdk2. Toxicology 278 (2), 224-228.

62. Yang, K. et al. (2006) Variations in cyclin D1 levels through the cell cycle determine the proliferative fate of a cell. Cell Div. 1, 32 .

63. Sundararaj, K.P. et al. (2004) Rapid shortening of telomere length in response to ceramide involves the inhibition of telomere binding activity of nuclear glyceraldehyde-3-phosphate dehydrogenase. J Biol.Chem 279 (7), 6152-6162.

64. Carujo, S. et al. (2006) Glyceraldehyde 3-phosphate dehydrogenase is a SET-binding protein and regulates cyclin B-cdk1 activity. Oncogene 25 (29), 4033-4042.

65. Jacquin, M.A. et al. (2013) GAPDH binds to active Akt, leading to Bcl-xL increase and escape from caspase-independent cell death. Cell Death.Differ. 20 (8), 1043-1054.

66. Kurdistani, S.K. (2014) Chromatin: a capacitor of acetate for integrated regulation of gene expression and cell physiology. Curr.Opin.Genet.Dev. 26C, 53-58.

67. Canto, C. et al. (2015) NAD(+) Metabolism and the Control of Energy Homeostasis: A

Balancing Act between Mitochondria and the Nucleus. Cell Metab 22 (1), 31-53.

68. Li, J. et al. (2015) Co-inhibition of polo-like kinase 1 and Aurora kinases promotes mitotic catastrophe. Oncotarget 6 (11), 9327-40.

69. Sur, S. and Agrawal, D.K. (2016) Phosphatases and kinases regulating CDC25 activity in the cell cycle: clinical implications of CDC25 overexpression and potential treatment strategies. Mol Cell Biochem 416 (1-2), 33-46.

70. Taguchi, N. et al. (2007) Mitotic phosphorylation of dynamin-related GTPase Drp1 participates in mitochondrial fission. J Biol.Chem 282 (15), 11521-11529.

71. Kashatus, D.F. et al. (2011) RALA and RALBP1 regulate mitochondrial fission at mitosis. Nat.Cell Biol. 13 (9), 1108-1115.

72. Peters, J.M. (2006) The anaphase promoting complex/cyclosome: a machine designed to destroy. Nat.Rev.Mol.Cell Biol. 7 (9), 644-656.

73. Jiang, Y. et al. (2014) PKM2 regulates chromosome segregation and mitosis progression of tumor cells. Mol Cell 53 (1), 75-87.

74. Wang, Z. et al. (2014) Cyclin B1/Cdk1 coordinates mitochondrial respiration for cellcycle G2/M progression. Dev.Cell 29 (2), 217-232.

75. Cai, L. and Tu, B.P. (2011) On acetyl-CoA as a gauge of cellular metabolic state. Cold Spring Harb.Symp.Quant.Biol. 76, 195-202.

76. Shi, L. and Tu, B.P. (2015) Acetyl-CoA and the regulation of metabolism: mechanisms and consequences. Curr.Opin.Cell Biol. 33, 125-131.

77. LeBleu, V.S. et al. (2014) PGC-1alpha mediates mitochondrial biogenesis and oxidative phosphorylation in cancer cells to promote metastasis. Nat Cell Biol 16 (10), 992-1003, 1-15. 
78. Kwon, S. et al. (2017) Obesity and aging diminish sirtuin 1 (SIRT1)-mediated deacetylation of SIRT3, leading to hyperacetylation and decreased activity and stability of SIRT3. J Biol Chem 292 (42), 17312-17323.

79. Aquilano, K. et al. (2010) Peroxisome proliferator-activated receptor gamma co-activator 1alpha (PGC-1alpha) and sirtuin 1 (SIRT1) reside in mitochondria: possible direct function in mitochondrial biogenesis. J Biol.Chem 285 (28), 21590-21599.

80. Kim, S.Y. et al. (2016) Forkhead Transcription Factor FOXO1 Inhibits Angiogenesis in Gastric Cancer in Relation to SIRT1. Cancer Res Treat 48 (1), 345-54.

81. Hardie, D.G. et al. (2012) AMPK: a nutrient and energy sensor that maintains energy homeostasis. Nat Rev Mol Cell Biol 13 (4), 251-62.

82. Porporato, P.E. et al. (2014) A mitochondrial switch promotes tumor metastasis. Cell Rep 8 (3), 754-66.

83. Walton, Z.E. et al. (2018) Acid Suspends the Circadian Clock in Hypoxia through Inhibition of mTOR. Cell 174 (1), 72-87.e32.

84. Blum, R. and Kloog, Y. (2014) Metabolism addiction in pancreatic cancer. Cell Death Dis 5, e1065.

85. Yu, L. et al. (2017) The Glycolytic Switch in Tumors: How Many Players Are Involved? J Cancer 8 (17), 3430-3440.

86. Akins, N.S. et al. (2018) Inhibition of Glycolysis and Glutaminolysis: An Emerging Drug Discovery Approach to Combat Cancer. Curr Top Med Chem 18 (6), 494-504.

87. Peyressatre, M. et al. (2015) Targeting cyclin-dependent kinases in human cancers: from small molecules to Peptide inhibitors. Cancers (Basel) 7 (1), 179-237.

88. Lis, P. et al. (2016) The HK2 Dependent "Warburg Effect" and Mitochondrial Oxidative

Phosphorylation in Cancer: Targets for Effective Therapy with 3-Bromopyruvate. Molecules

21 (12), 1730.

89. Icard, P. et al. (2018) Extracellular Citrate and Cancer Metabolism-Letter. Cancer Res 78

(17), 5176.

90. Ren, J.G. et al. (2017) Citrate Suppresses Tumor Growth in Multiple Models through Inhibition of Glycolysis, the Tricarboxylic Acid Cycle and the IGF-1R Pathway. Sci Rep 7 (1), 4537.

91. Branco, A.F. et al. (2016) Ketogenic diets: from cancer to mitochondrial diseases and beyond. Eur J Clin Invest 46 (3), 285-98.

92. Zdralevic, M. et al. (2018) Disrupting the 'Warburg effect' re-routes cancer cells to OXPHOS offering a vulnerability point via 'ferroptosis'-induced cell death. Adv Biol Regul $68,55-63$.

\section{Figure 1. The Metabolism of Cancer Cells}

Cancer cells depend on aerobic glycolysis, glutaminolysis, and $\beta$-oxidation of fatty acids, for the synthesis of ribose, glucosamine, polyamines, lipids, and cholesterol. The Warburg effect is related to pyruvate dehydrogenase (PDH) inhibition by PDK1 and LDH-5 activation and produces lactate, which is expulsed by MCT (green pathway). A bottleneck is produced at the end of glycolysis by the dimeric PKM2 isoform producing an accumulation of metabolites upstream. Thus, glucose 6-phosphate (G6P) sustains the pentose phosphate pathway that 
supports the ribose biosynthesis required for nucleotide synthesis. 3 phosphoglycerate (3PG) feeds the serine-methionine synthesis that sustains nucleotide, glutathione, and polyamines formation (purple pathway). Other metabolites sustain metabolism such as fructose 6phosphate (F6P) that enters the glucosamine pathway and supports glycosylation of proteins or dihydroxyacetone phosphate (DHAP) sustaining the triglyceride synthesis entering the lipid pathway.

In mitochondria, acetyl-CoA derives from both the degradation of the $\beta$-oxidation of fatty acids (Acyl-CoA) and from the glutamine pathway producing glutamate (Glu) sustaining $\alpha$ ketoglutarate ( $\alpha$-Keto) entering the tricarboxylic acid cycle (TCA). An excess of mitochondrial citrate is exported to the cytosol where it is catalyzed by acetyl-coA carboxylase (ACLY) into oxaloacetate (OAA) and acetyl-CoA. This later metabolite sustains the lipid and mevalonate pathways.

Acetyl-CoA, ACC: acetyl-coA carboxylase, ACLY: ATP citrate lyase, ALDO: Aldolase, 1,3 BPG: 1, 3-Bisphosphogylcerate, DHAP: dihydroxyacetone phosphate, FA: Fatty acids, F6P: fructose 6-phosphate, F1,6P: fructose-1,6-bisphosphate, F2,6BP: fructose-2,6-biphosphate, G: glucose, G6P: glucose 6-phosphate, G6PDH: glucose-6-phosphate dehydrogenase, GA3P: glyceraldehyde3-phosphate, GAPDH: glyceraldehyde 3-phosphate dehydrogenase, Glu: glutamate, Gln: glutamine, GFAT1: glutamine-fructose-6-phosphate transaminase 1, GLS1: glutamine synthase1, GLUT1: membrane glucose transporter 1, G3P: glyceraldehyde-3phosphate, HK2: hexokinase 2, HMG-CoA: 3-Hydroxy-3-methylglutaryl-CoA, $\alpha$-KG: $\alpha$-keto, LDH-5: lactate dehydrogenase 5, MCT: monocarboxylate transporter, $\mathrm{NAD}^{+}$: nicotinamide adenine dinucleotide, NADPH, $\mathrm{H}^{+}$: nicotinamide adenine dinucleotide phosphate, OAA: Oxaloacetate, PDH: pyruvate dehydrogenase, PDK1: pyruvate dehydrogenase kinase1, PEP: phosphoenolpyruvate, PFK1: phosphofructokinase1, PFKFB3: 6-phosphofructo-2kinase/fructose-2,6-biphosphatase 3, 2-PG: 2 phosphoglycerate, 3-PG: 3 phosphoglycerate, PGK1: phosphoglycerate kinase1, PKM2: embryonic pyruvate kinase, PDH: pyruvate dehydrogenase, PEP: phosphoenolpyruvate, R5P: ribose 5-phosphate, TKL1: transketolase1, TPI: triosephosphate isomerase, UDP-GlcNAc: uridine diphosphate $\mathrm{N}$-acetylglucosamine 


\section{Figure 2. A Model of Cell Cycle Progression}

A: Cell cycle progression is controlled by periodic activation of major cyclin-CDKs. Mitogenic factors promote synthesis of D-type cyclins which form complexes with CDK4/6 resulting in phosphorylation of pRB. E2F1 repression is relieved and progression towards a G1/S transition allowed. Cyclin E regulates S phase entry and cyclin A progression to $\mathrm{S}$ and G2 phases. Phosphorylation of E2F1 by cyclin A-CDK2 arrests DNA synthesis.

B: $\mathrm{SCF}^{\beta-\operatorname{TrCP}}$ positively regulates $\mathrm{APC} / \mathrm{C}^{\mathrm{Cdc} 20}$ degrading cyclin $\mathrm{A}$ and ensuring $\mathrm{G} 2 / \mathrm{M}$ transition. Cyclin $\mathrm{B}-\mathrm{CDK} 1$ triggers mitosis and $\mathrm{APC} / \mathrm{C}^{\mathrm{Cdh} 1}$ regulates exit of mitosis. Phosphatase $C d c 25 A$ is required for progression from $\mathrm{G} 1$ to $\mathrm{S}$ phase. In contrast to Cdc25C, WEE1 inhibits CDK1 and G2 checkpoints. PFKFB3 and GLS1 are activated in mild to late $\mathrm{G} 1$ due to inactivation of $\mathrm{APC} / \mathrm{C}^{\mathrm{Cdh} 1}$. Their activation helps bypass the restriction checkpoint. GLS1 is degraded in late mitosis when $\mathrm{APC} / \mathrm{C}^{\mathrm{Cdh} 1}$ is again active, this is in contrast to PFKFB3 where degradation begins at the onset of mitosis as it is also degraded by $\mathrm{SCF}^{\beta-\operatorname{TrCP}}$.

C: Sequential activation of metabolic enzymes adequately links biosynthesis with a period of interphase (for example, activation of GLS1, G6PD, and TKL1 which support DNA synthesis) while several enzymes also have nuclear functions promoting cell cycle and proliferation.

APC/C (Anaphase-promoting complex/cyclosome), ALDO: Aldolase, Cdc25: Cell Division Cycle 25, E2F1: E2F transcription factor 1, GAPDH: glyceraldehyde 3-phosphate dehydrogenase, GLS1: glutamine synthase1, HK2: hexokinase 2, PFKFB3: 6-phosphofructo2-kinase/fructose-2,6-biphosphatase 3, PKM2i: dimeric or monomeric embryonic pyruvate kinase, PKM2a: tetrameric active isoform, pRB: retinoblastoma suppressor protein, SCF (Skp1/ Cullin/F-box), STAT3: Signal transducer and activator of transcription 3, TKL1: transketolase 1

Figure 3, Key Figure:-Periodical Activation of Metabolic Enzymes and Cyclin-CDKs Promote Cell Cycle Progression.

Cyclic activation of cyclin-CDKs regulate cell cycle progression, for example, cyclins D/CDK4-6 in G1 phase, cyclin E/CDK2 in S phase, cyclin A/CDK2-1 in G2 phase, and 
Cyclin B/CDK1 in M phase. Glycolytic enzymes (HK2, PKM2 inactive form), PFKFB3, and histone acetyl transferases (HATs) mainly support protein synthesis in G1 phase. Then, ALDO promotes the progression in S phase. During the G2 phase, PKM2 active form and GAPDH are activated. Glutaminolysis regulated by GLS1 and the pentose phosphate pathway (PPP) by G6PDH and TKL sustain DNA replication in S phase and lipid synthesis in G2 phase, respectively. The sequential activations of these metabolic enzymes are coordinated with those of cyclins by two processes, (i) the periodical translocation of a few enzymes to the nucleus where they promote gene expression of cell cycle activators (CCDN1 encoding cyclin D1) /or oncogenes (c-Myc), (ii) the periodic degradation of cyclins and metabolic enzymes by ubiquitin complexes.

\section{Glossary}

Crabtree effect: in response to a huge consumption of glucose, cancer cells similar to yeast, may reversibly down-regulate oxygen consumption and increase lactate production. This metabolic effect should be protective against overproduction of ROS. It could reinforce the Warburg effect, contributing to cancer cell growth.

Epigenetic: chromatin accessibility is regulated by dynamic and reversible epigenetic modifications such as DNA methylation, histone methylation, and acetylation which render chromatin less or more accessible to transcription. Histone deacetylation is required for transcriptional repression and chromatin compaction before $M$ phase entry.

Mitophagy: a process whereby lysosomes degrade mitochondria in response to damage caused by various phenomena such as stress, ROS, and senescence. This turnover process is essential for maintaining the integrity of the cell. 
Reverse Warburg effect: ROS stimulate an aerobic glycolysis in cancer associated fibroblasts (CAFs) which can expel lactate into the microenvironment. Lactate can be takenup by some cancer cells to be recycled in the TCA cycle, sustaining their oxidative metabolism. This 'lactate shuttle' can drive tumor progression, metastasis, and even drug resistance.

Ubiquitin: ubiquitination is an enzymatic post-translational modification by which ubiquitin is attached to lysine residues on a protein by ubiquitin ligases (E3s) for the degradation of the protein by the proteasome. Ubiquitination requires three types of enzyme: ubiquitin-activating enzymes (E1s), ubiquitin-conjugating enzymes (E2s), and ubiquitin ligases (E3s). 


\section{Box 1. Cell Cycle Events}

In proliferative eukaryotic cells, the series of events which lead to DNA replication and cell division are ordered and divided into three periods: (i) interphase, (ii) mitosis (M); and (iii) cytokinesis. Interphase, typically represents $90 \%$ of the total time required for the cell cycle, and proceeds in three phases (G1, S, and G2). The irreversibility of the cell cycle is controlled by three main checkpoints, the first is the G1 checkpoint, also known as the restriction checkpoint; followed by the G2/M checkpoint; and finally the metaphase checkpoint, also known as the spindle checkpoint.

G1 phase is the growth phase, where cells make provision of metabolites while most of the biosynthesis occurs. It is the longest phase of the whole cycle, but also the most variable in duration. Cells synthesize proteins and prepare DNA replication occurring in S phase. During G2 phase, cells enhance their de novo lipid synthesis required for membrane formation, and ensure that everything is ready to initiate the mitotic process.

$\mathrm{M}$ phase is a relatively brief period of the cycle consisting of spindle assembly of chromosomes, followed by their segregation, and nuclear division. It is divided into subphases: prophase (nuclear envelope breakdown, chromosome condensation, and association to mitotic spindle), prometaphase (alignment of replicated chromosomes on the spindle), metaphase (bi-orientation of all separated chromosomes on the central plate), anaphase (cleavage of cohesin rings and separation of sister chromatids to opposite poles of the spindle), and telophase (reassembly of envelopes around the daughter nuclei). Telophase is, generally, immediately followed by cell division (cytokinesis) which divides nuclei, cytoplasm, organelles, and the cell membrane and finally separates two daughter cells with identical sets of chromosomes, and roughly equal shares of cellular components. 
Cell cycle progression is complex and highly regulated by activators (cyclin-CDKs) and inhibitors degrading cyclin or inhibiting the sequential activation of cyclin-CDKs. Errors occurring at different steps of the cell cycle can lead to apoptosis or cause genetic instability promoting cancer aggressiveness. Abnormalities in cytokinesis can lead to the occurrence of cells with multiple nuclei, a process called endoreplication.

\section{Box 2. Metabolic strategies aiming at counteracting cancer cell metabolism}

The inhibition of enzymatic reactions involved in the metabolism of cancer cells could be reversed by the fact that some cells can survive by developing alternative metabolic pathways and/or promoting autophagy. Thus, other metabolic approaches should be considered for inhibiting cancer cell growth: (i) the activation of mitochondria could reverse the Warburg effect and promote apoptosis by favoring ROS overproduction. This reversal might be attempted by the inhibition of lactate production or recycling, by increasing PKM2 tetrameric activity and/or promoting PDH activity. However, the reactivation of OXPHOS potentially favors resistance and/or metastases of some cell-clones [77, 82, 92]. Thus, lethal production of ROS could favor the inhibition of the cysteine transporter, because cysteine is involved in the biosynthesis of glutathione, which has a protective role against ROS [92]. It follows that depriving cancer cells of an essential molecule can be a successful strategy as it has been demonstrated to be so in the past for treatment of lymphoblastic leukemia; (ii) the promotion of a microenvironment hostile to cancer development could be favored by counteracting the $\mathrm{pH}$ using inhibitors of membrane transporters and/or alkaline salts [9]. Of note, sodium citrate at high doses inhibits glycolysis, arrests growth of various cultured cancer cells, and increases sensibility to chemotherapy in vitro and in vivo studies $[89,90]$. 


\section{Glucose}

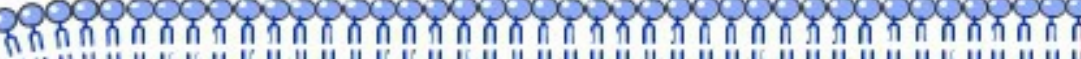

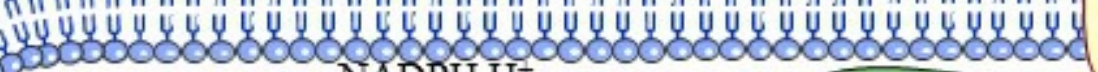

Apovo9o $\mathrm{NADPH}, \mathrm{H}^{+}$

Ribulose-5P Oxidative stage

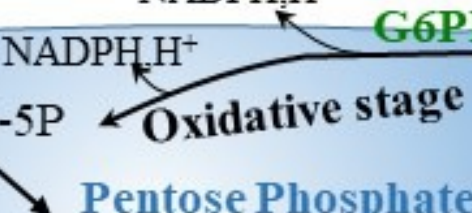

$\mathrm{R}-5 \mathrm{P}$ Xy-5P pathway
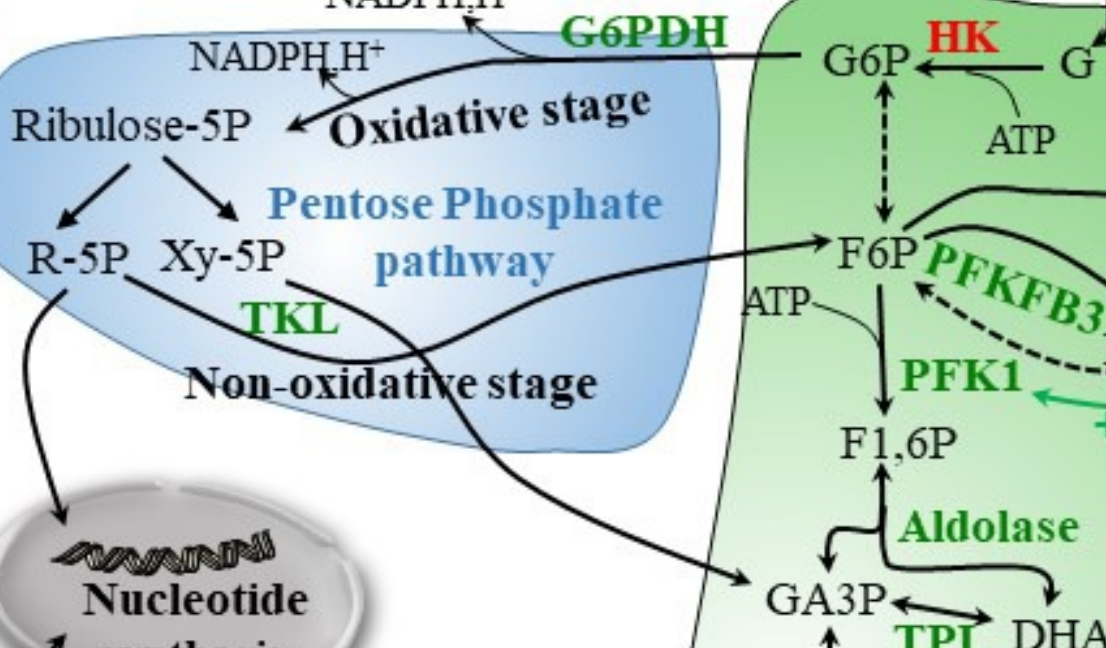

\section{2}

ATP
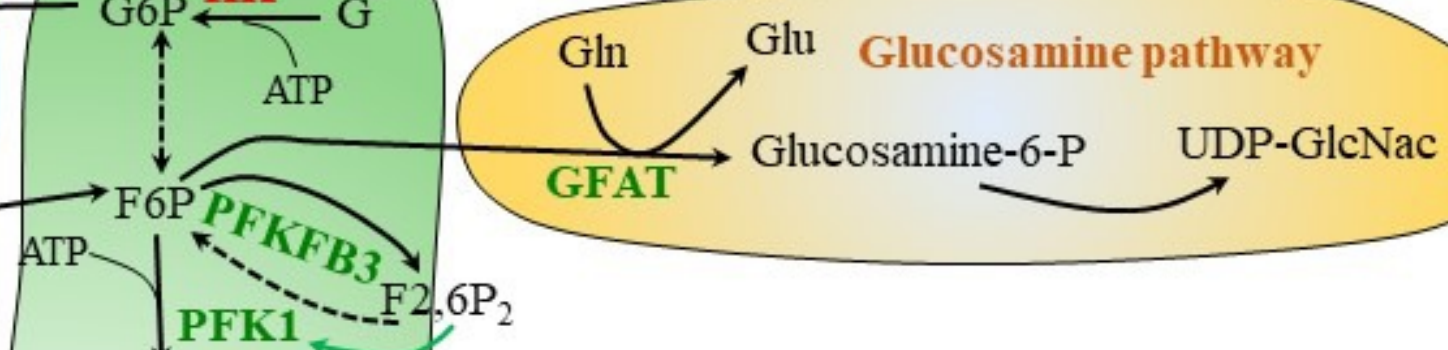

glycosylation

synthesis

Folate

vnthesis
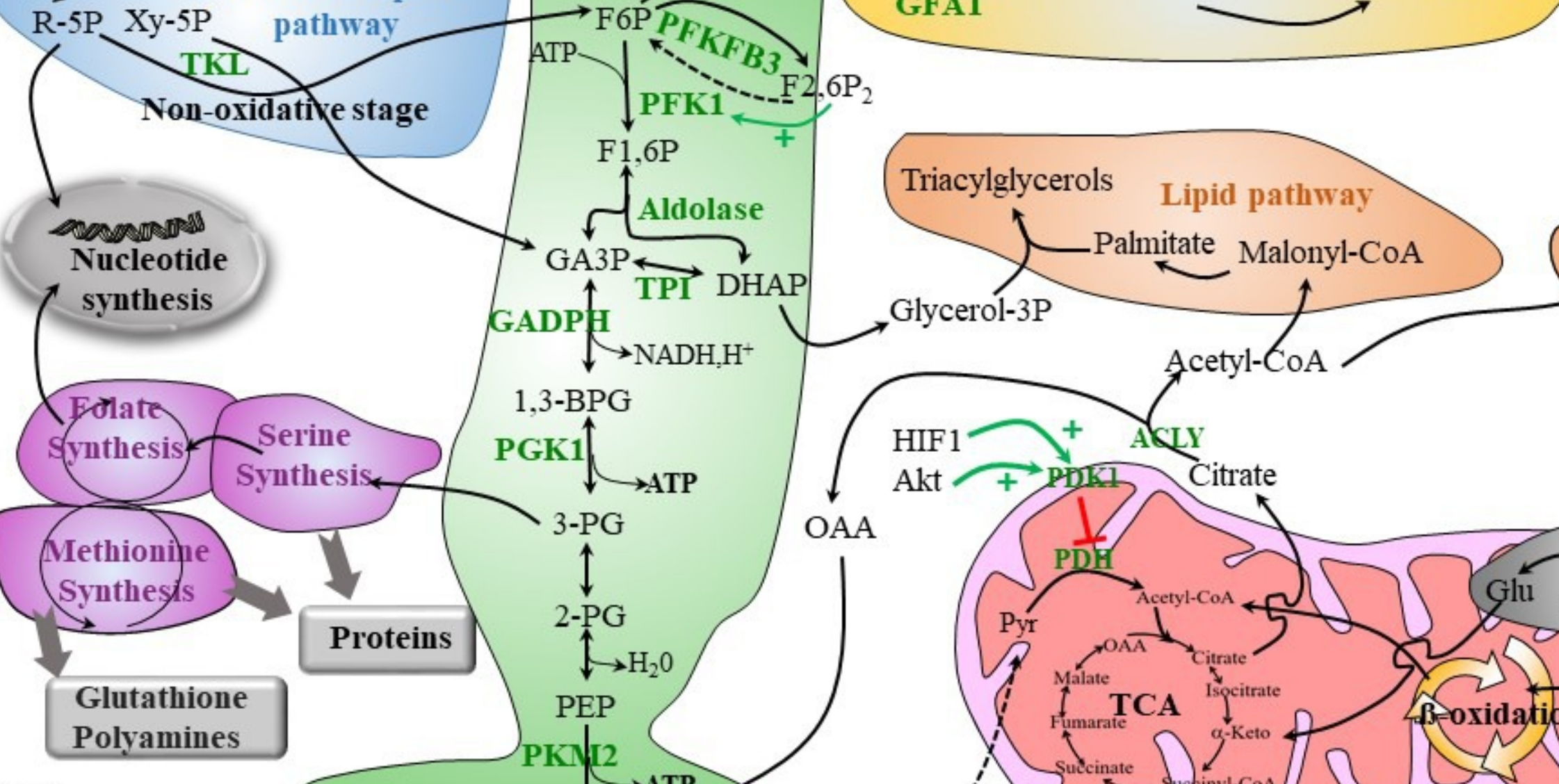

Lipid pathway 


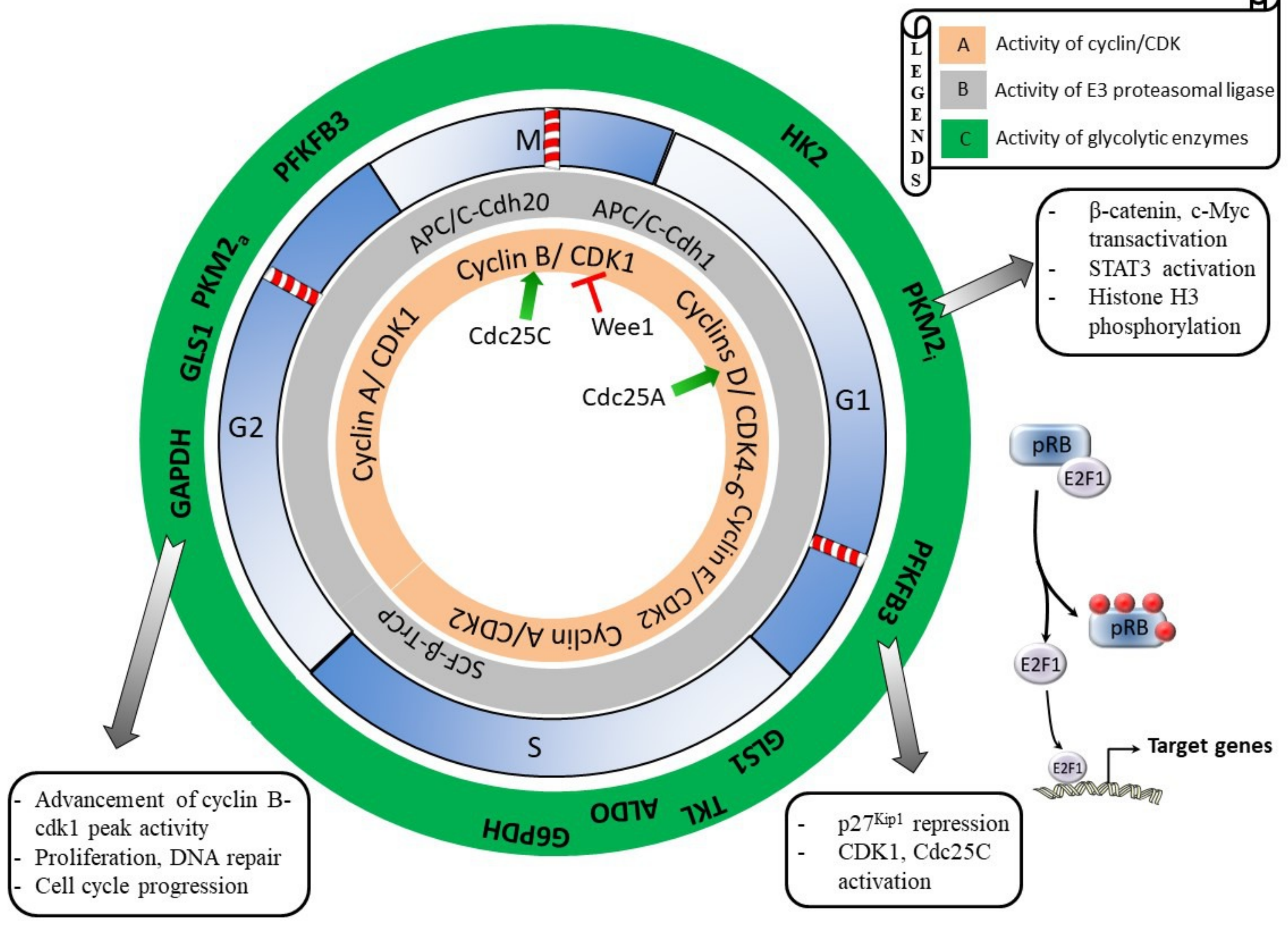

